\title{
Perfil sociodemográfico, situação de saúde e práticas de autocuidado dos idosos com Doença Crônica Não Transmissível em um município Amazônico
}

Sociodemographic profile, health situation and self-care practices of elderly with Chronic
Noncommunicable Disease in Amazonian municipalitiy

Perfil sociodemográfico, situación de salud y prácticas de autocuidado de los ancianos con Enfermedad Crónica No Transmisible en un municipio Amazónico

Deyvylan Araujo Reis ${ }^{1 *}$, Maxwell Arouca da Silva1.

\section{RESUMO}

Objetivo: Caracterizar o perfil sociodemográfico, situação de saúde e as práticas de autocuidado em idosos cadastrados na rede básica de saúde do município de Coari, Estado do Amazonas. Métodos: Estudo exploratório, descritivo e transversal com abordagem quantitativa, com população de 340 idosos. A coleta de dados ocorreu no mês de janeiro a junho de 2015, por meio de formulário sociodemográfico, situação de saúde e práticas de autocuidado. Resultados: Predominância do sexo feminino (59,0\%), faixa etária de 60 a 69 anos $(28,0 \%)$ e baixo nível de escolaridade $(87,0 \%)$. Verificou-se que as doenças do aparelho circulatório apresentam maior frequência (46,7\%) entre as Doenças Crônicas Não Transmissíveis, especificamente com a Hipertensão arterial sistêmica entre os idosos jovens $(47,0 \%)$. Conclusão: $O$ conhecimento dos profissionais integrantes da equipe Estratégia Saúde da Família pode ter bases concretas para planejamento de ações em saúde a partir das necessidades desses indivíduos.

Palavras-chave: Perfil de saúde, Idoso, Doença Crônica.

\begin{abstract}
Objective: To characterize the sociodemographic profile, health status and self-care practices of the elderly registered in the primary healthcare network in the municipality of Coari, Amazonas State, Brazil. Methods: Exploratory, quantitative, descriptive, crosscut study with a population of 340 elderly people. Data collection was carried out between January and June of 2015 , by means of a sociodemographic formulary, health status and self-care practices. Results: Females prevailed (59,0\%), age ranged between 60 and 69 years (28,0\%), and low level of schooling (87\%). It was evidenced that circulatory system diseases were more frequent $(46,7 \%)$ from the Noncommunicable Diseases, specifically the Arterial Systemic Hypertension among the young seniors $(47,0 \%)$. Conclusion: It was concluded that professionals' knowledge from the Family Health Strategy team may be concretely grounded in order to plan healthcare actions in view of those individuals' needs.
\end{abstract}

Keywords: Health profile, Elderly person, Chronic Disease.

${ }^{1}$ Instituto de Saúde e Biotecnologia (ISB) da Universidade Federal do Amazonas (UFAM), Coari-Amazonas.

*E-mail: deyvylan@ufam.edu.br

Financiamento da Fundação de Amparo à Pesquisa do Estado do Amazonas (FAPEAM). PIB.0058/2014 


\section{RESUMEN}

Objetivo: Caracterizar el perfil sociodemográfico, situación de salud y las prácticas de autocuidado en ancianos catastrados en la red básica de salud del municipio de Coari, Estado de Amazonas. Métodos: Estudio exploratorio, descriptivo y transversal con abordaje cuantitativo, con población de 340 ancianos. La recolección de datos ocurrió en el mes de enero a junio de 2015, por medio de formulario sociodemográfico, situación de salud y prácticas de autocuidado. Resultados: Predominancia del sexo femenino (59,0\%), grupo de edad de 60 a 69 años (28,0\%) y bajo nivel de escolaridad (87,0\%). Se verificó que las enfermedades del aparato circulatorio presentan mayor frecuencia (46,7\%) entre las Enfermedades Crónicas no Transmisibles, específicamente con la Hipertensión arterial sistémica entre los ancianos jóvenes (47,0\%). Conclusión: El conocimiento de los profesionales integrantes del equipo Estrategia Salud de la Familia puede tener bases concretas para la planificación de acciones en salud a partir de las necesidades de esos individuos.

Palabras clave: Perfil de salud, Ancianos, Enfermedad Crónica.

\section{INTRODUÇÃO}

O Brasil é um país em desenvolvimento e com aumento progressivo da proporção de idosos, segundo o Instituto de Brasileiro de Geografia e Estatística, o qual constatou que, no ano de 2005 a 2015, houve um aumento de $9,8 \%$ para $14,3 \%$ de indivíduos com idade igual e superior a 60 anos. Estima-se que no ano de 2050, o Brasil deverá triplicar em número absoluto esse grupo populacional, alcançando, assim, cerca de 66,5 milhões de idosos (IBGE, 2016; FIGUEIREDO AH, 2016).

Essa expansão populacional resultará numa demanda crescente por atenção à saúde em decorrência do aumento das taxas de incidência das Doenças Crônicas Não Transmissíveis (DCNT) na população envelhecida.

O aumento da taxa de prevalência da DCNT na pessoa idosa pode acarretar importantes implicações para a saúde como a utilização excessiva dos serviços de saúde, institucionalizações (muitas vezes desnecessárias) e diversas incapacidades ou limitações físicas e cognitivas para realizar as tarefas do cotidiano (DUCAN BB, et al., 2012; MALTA DC, et al., 2017). O aparecimento de uma DCNT implica na necessidade de modificação no hábito de vida do indivíduo, ou seja, desenvolver as práticas de autocuidado como forma de controlar a própria enfermidade.

As DCNTs caracterizam-se por uma enfermidade de longo prazo, com duração de três meses ou mais, com evolução gradual dos sintomas e da improbabilidade de resolução espontânea, sendo potencialmente incapacitantes (CHEEVER KH, et al., 2016).

Em decorrência das DCNTs não serem emergenciais, provocam impacto econômico e social aos idosos, às famílias e à comunidade. Com isso, são responsáveis por $72,4 \%$ das causas de óbitos no Brasil, com destaque para as doenças do aparelho circulatório, neoplasia, Diabetes Mellitus e Doença Respiratória Crônica (DUCAN BB, et al., 2010).

Esse grupo de DCNTs pode repercutir na pessoa idosa, em vários domínios de sua vida, afetar as suas atividades de vida diária e, muitas vezes, abrir portas para outras comorbidades. É necessário o acompanhamento dos idosos na atenção básica de saúde para que se possa definir o perfil dessas enfermidades e o preparo dos profissionais da atenção primária para uma abordagem multiprofissional e interdisciplinar (ROCHA FCV, et al., 2011).

O conhecimento da população atendida na área de abrangência da Unidade Básica de saúde em relação ao perfil sociodemográfico, situação de saúde e suas práticas de autocuidado é de suma importância, pois possibilita detectar as intervenções bem-sucedidas e formular objetivos e metas para o setor, a fim de gerar melhorias na assistência prestada. Sendo assim, foi estabelecida a seguinte questão de pesquisa: Qual é o perfil sociodemográfico, situação de saúde e as práticas de autocuidado em idosos atendidos na rede básica de saúde do município de Coari, Amazonas? 
Diante do exposto, o objetivo deste estudo foi caracterizar o perfil sociodemográfico, situação de saúde e as práticas de autocuidado em idosos cadastrados na rede básica de saúde do município de Coari, Estado do Amazonas.

\section{MÉTODO}

Estudo exploratório, descritivo e transversal com abordagem quantitativa, realizado na área urbana do município de Coari, no interior do Estado do Amazonas, na região norte do Brasil. O município é a quinta cidade mais populosa do Estado, o qual de acordo com os dados do Censo demográfico no ano de 2010, possuía 75.965 mil habitantes (IBGE, 2011), dos quais 2.931 são idosos cadastrados e atendidos pela Estratégia Saúde da Família (ESF).

A população da pesquisa foi constituída por 340 idosos com idade igual ou superior a 60 anos, de ambos os sexos, cadastrados nas 11 equipes de saúde da família. Este tamanho de amostra permitiu estimativas com margem de erro de $\pm 0,05 \%$ e intervalo de confiança de $95 \%$, onde 1,96 foi o escore da curva normal para o intervalo de $95 \%$. Contudo, obteve-se o número amostral com o qual se pretendia trabalhar neste estudo, ou seja, de $12 \%$ da população de idosos.

Este estudo foi desenvolvido através de amostragem por conglomerado por área, a escolha do indivíduo em cada Unidade Básica de Saúde (UBS). Dessa forma, o levantamento desses participantes do estudo deuse através de uma visita inicial às UBSs, com o intuito de reunir-se com os agentes comunitários de saúde, a fim de identificar os idosos com diagnóstico médico de DCNT, além do acesso aos cadastros dos indivíduos acompanhados pela ESF.

Após essa identificação, os dados coletados foram digitados em uma planilha do programa de Microsoft Excel (versão 2010), os gerontes atendidos em cada UBS do município.

Para a identificação dos idosos, foi realizado um sorteio através de um programa chamado Microsoft Excel 7 de Sistema de sorteio de planilha 1.0, com o objetivo de selecionar os indivíduos. Após esta etapa, foi elaborado um cronograma com identificação de nome e endereço para dar início à coleta de dados.

$\mathrm{Na}$ visita domiciliar aos idosos selecionados para o estudo, foi esclarecida a importância da sua participação na pesquisa. Após um breve esclarecimento, todos os 340 indivíduos idosos concordaram em participar da pesquisa e assinaram o Termo de Consentimento Livre e Esclarecido, de acordo com a resolução 466/12 do Conselho Nacional de Saúde, relativa à pesquisa envolvendo seres humanos (CNS, 2015).

A coleta de dados foi realizada no domicílio do participante do estudo, no período de janeiro a junho de 2015, mediante aplicação de formulário, construído especificamente para este estudo, com questões que atendem as variáveis demográficas (idade e sexo) socioeconômicas (idade, cor autodeclarada, estado conjugal, escolaridade, profissão e renda mensal da família), situação de saúde (apresenta alguma DCNT) e as práticas de autocuidado (número de refeições diárias, tabagismo, consumo de bebida alcoólica, etilismo, atividade física).

Os dados e as informações coletadas foram registrados em uma planilha do programa Microsoft Excel 2010, utilizando-se para análise estatística o programa Statistical Package for the Social Sciences (SPSS versão 20.0) e as variáveis apresentadas de forma descritiva, mediante frequência simples e percentual. A presente pesquisa foi aprovada pelo Comitê de Ética e Pesquisa (CEP) da Universidade Federal do Amazonas conforme a resolução $n^{\circ}$ 466/2012, sob o CAAE $n^{\circ}$ 35573614.3.000.5020.

\section{RESULTADOS}

Dos 340 participantes do estudo, 59,1\% eram do sexo feminino. Predominaram idosos na faixa etária de 60 a 69 anos (46,8\%), com baixo nível de escolaridade entre analfabeto $(44,1 \%)$ e um a cinco anos de estudo $(43,2 \%)$, tem cor autodeclarado de pardo $(71,8 \%)$, casados ou união estável $(56,2 \%)$. Tinham como ocupação aposentado $(77,6 \%)$ e declaram possuir renda mensal entre dois a três salários mínimos (Tabela 1). 
Tabela 1 - Caracterização das variáveis sociodemográficas, segundo o sexo dos idosos. $\mathrm{n}=340$. Coari - AM, 2015.

\begin{tabular}{|c|c|c|c|}
\hline Variáveis sociodemográficas & Feminino N (\%) & Masculino N (\%) & Total N (\%) \\
\hline \multicolumn{4}{|l|}{ Idade } \\
\hline 60 a 69 & $96(28,2)$ & $63(18,5)$ & $159(46,8)$ \\
\hline 70 a 79 & $64(18,8)$ & $48(14,1)$ & $112(32,9)$ \\
\hline $80 \mathrm{ou}+$ & $41(12,1)$ & $28(8,2)$ & $69(20,3)$ \\
\hline \multicolumn{4}{|l|}{ Cor autodeclarada } \\
\hline Parda & $151(44,4)$ & $93(27,4)$ & $244(71,8)$ \\
\hline Branca & $34(10,0)$ & $28(8,2)$ & $62(18,2)$ \\
\hline Negra & $16(4,7)$ & $18(5,3)$ & $34(9,7)$ \\
\hline \multicolumn{4}{|l|}{ Estado conjugal } \\
\hline Casado (a) / União Estável & $98(28,8)$ & $93(27,4)$ & $191(56,2)$ \\
\hline Viúvo (a) & $72(21,2)$ & $13(3,8)$ & $85(25,0)$ \\
\hline Solteiro/Separado (a) & $31(9,1)$ & $33(9,7)$ & $64(18,8)$ \\
\hline \multicolumn{4}{|c|}{ Escolaridade (em anos de estudo) } \\
\hline 0 & $79(23,2)$ & $71(20,9)$ & $150(44,1)$ \\
\hline 1 a 5 & $95(27,9)$ & $52(15,3)$ & $147(43,2)$ \\
\hline 6 a 11 & $18(5,3)$ & $8(2,4)$ & $26(7,6)$ \\
\hline$\geq 12$ & $9(2,6)$ & $8(2,4)$ & $17(5,0)$ \\
\hline \multicolumn{4}{|l|}{ Ocupação } \\
\hline Aposentado & $156(45,9)$ & $108(31,8)$ & $264(77,6)$ \\
\hline Autônomo & $26(7,6)$ & $26(7,6)$ & $52(15,3)$ \\
\hline Pensionista & $11(3,2)$ & $3(0,9)$ & $14(4,1)$ \\
\hline Desempregado & $8(2,4)$ & $2(0,6)$ & $10(2,9)$ \\
\hline \multicolumn{4}{|c|}{ Renda mensal familiar (salário mínimo ${ }^{\dagger}$ ) } \\
\hline 0 a 1 & $75(22)$ & $55(16)$ & $130(38)$ \\
\hline 2 a 3 & $113(33)$ & 75 (22) & $188(55)$ \\
\hline 4 a 5 & $13(4)$ & $9(3)$ & $22(7)$ \\
\hline
\end{tabular}

Nota: †Salário mínimo vigente no valor de 788 reais. Fonte: Reis DA, Silva MA, 2019.

Com relação às DCNTs dos idosos, verificou-se que as doenças do aparelho circulatório $(46,7 \%)$ apresentam maior frequência (Figura 1). Dentre essas doenças, a mais citada foi a HAS (58,2\%) (Tabela 2). Quanto às doenças endócrinas, nutricionais e metabólicas $(36,0 \%)$, constatou-se que foi ao segundo grupo de DCNT mais citado, especificamente a dislipidemia $(25,3 \%)$ e DM $(24,1 \%)$.

Figura 1 - Distribuição de frequência das Doenças Crônicas Não Transmissíveis, segundo o capítulo da CID 10. $n=340$. Coari - AM, 2015.

Doenças do aparelho circulatório (DAC)

Doenças endócrinas, nutricionais e metabólicas (DENM)

Doenças do sistema osteomuscular e do tecido conjuntivo (DO)

Doença do olho e anexos (DOA)

$2,8 \%$

$$
\text { Neoplasias (NEO) } \quad 0,9 \%
$$

Transtornos mentais e comportamentais (TMC) | 0,4\%

Doenças do aparelho respiratório (DAR) | 0,4\%

Nota: Todos os idosos relataram mais de uma DCNT. Fonte: Reis DA, Silva MA, 2019. 
Em referência ao tempo relacionado ao conhecimento da HAS, dislipidemia e DM, o estudo aponta como mais frequente, maior ou igual a seis anos informado pelos participantes da pesquisa.

No que refere aos antecedentes familiares com comorbidades crônicas, a maioria dos idosos relatou que possuía familiares com alguma DCNT, parentesco de primeiro grau $(17,0 \%)$, o que confirma que a hereditariedade e outros fatores são predisponentes para o desenvolvimento da doença, apesar de a grande parcela dos participantes do estudo referirem que desconheciam história familiar de DCNT entre seus familiares.

Com relação à prática para o autocuidado, o estudo revelou que a maioria dos idosos realizavam de quatro a cinco refeições por dia, seguindo-se por seis a sete refeições por dia (Tabela 3). Quanto ao tabagismo e etilismo, o estudo revelou que os participantes não têm o hábito de fumar $(299 / 87,9 \%)$ e consumir bebidas alcoólica $(321 / 94,4 \%)$ em todas as faixas etárias.

Quanto à prática de atividade física regular, a pesquisa mostrou que grande parte dos participantes tem o costume de realizar exercício físico (179/52,6\%), em uma frequência de três a quatro vezes por semana, principalmente entre os idosos mais jovens $(94 / 27,6 \%)$.

Tabela 2- Distribuição de frequência das comorbidades crônicas, segundo a faixa etária. n=340. Coari - AM, 2015.

\begin{tabular}{lcccc}
\hline \multirow{2}{*}{ Comorbidades crônicas } & \multicolumn{3}{c}{ Faixa etária (anos) } & Total \\
\cline { 2 - 4 } & $\begin{array}{c}\mathbf{6 0} \text { a } \mathbf{~ 6 9} \\
\mathbf{N ~ ( \% )}\end{array}$ & $\begin{array}{c}\mathbf{7 0} \text { a 79 } \\
\mathbf{N}(\%)\end{array}$ & $\begin{array}{c}\mathbf{2} \mathbf{8 0} \\
\mathbf{N}(\%)\end{array}$ & $\mathbf{N}(\%)$ \\
\hline Hipertensão Arterial Sistêmica & & & & \\
(HAS) & $93(47,0)$ & $82(41,4)$ & $23(11,6)$ & $19(100,0)$ \\
Dislipidemia & $42(48,8)$ & $28(32,6)$ & $16(18,6)$ & $86(100,0)$ \\
Diabetes Mellitus & $34(41,5)$ & $28(34,1)$ & $20(24,4)$ & $82(100,0)$ \\
Artrite reumatoide & $5(11,6)$ & $28(65,1)$ & $10(23,3)$ & $43(100,0)$ \\
Glaucoma & $0(0,0)$ & $8(61,5)$ & $5(38,5)$ & $13(100,0)$ \\
Osteoporose & $1(10,0)$ & $3(30,0)$ & $6(60)$ & $10(100,0)$ \\
Artrose & $0(0,0)$ & $2(28,6)$ & $5(71,4)$ & $7(100,0)$ \\
Asma & $1(50,0)$ & $1(50,0)$ & $0(0,0)$ & $2(100,0)$ \\
Transtorno depressivo & $0(0,0)$ & $1(50,0)$ & $1(50,0)$ & $2(100,0)$ \\
Mal de Parkinson & $0(0,0)$ & $0(0,0)$ & $1(100,0)$ & $1(100,0)$ \\
Insuficiência Cardíaca & $0(0,0)$ & $0(0,0)$ & $1(100,0)$ & $1(100,0)$ \\
Congestiva & &
\end{tabular}

Nota: Todos os idosos relataram mais de uma DCNT. Fonte: Reis DA, Silva MA, 2019.

Tabela 3 - Distribuição de frequência das práticas de autocuidado dos idosos com Doenças Crônicas Não Transmissíveis, segundo a faixa etária. n=340. Coari - AM, 2015.

\begin{tabular}{|c|c|c|c|c|}
\hline \multirow[b]{2}{*}{ Práticas de autocuidado } & \multicolumn{3}{|c|}{ Faixa etária (anos) } & \multirow[b]{2}{*}{ Total N (\%) } \\
\hline & 60 a $69 \mathrm{~N}(\%)$ & 70 a $79 \mathrm{~N}(\%)$ & $\geq 80 \mathrm{~N}(\%)$ & \\
\hline \multicolumn{5}{|l|}{ Tabagismo } \\
\hline Não & $136(40,0)$ & $102(30,0)$ & $61(17,9)$ & $299(87,9)$ \\
\hline Sim & $23(6,8)$ & $10(2,9)$ & $8(2,4)$ & $41(12,1)$ \\
\hline Total & $159(46,8)$ & $112(32,9)$ & $69(20,3)$ & $340(100)$ \\
\hline \multicolumn{5}{|l|}{ Etilismo } \\
\hline Não & $145(42,6)$ & $108(31,8)$ & $67(19,7)$ & $321(94,4)$ \\
\hline Sim & $14(4,1)$ & $4(1,2)$ & $2(0,6)$ & $19(5,6)$ \\
\hline Total & $159(46,8)$ & $112(32,9)$ & $69(20,3)$ & $340(100,0)$ \\
\hline \multicolumn{5}{|l|}{$\begin{array}{l}\text { Prática de atividade física } \\
\text { regular }\end{array}$} \\
\hline Não & $65(19,1)$ & $51(15,0)$ & $45(13,2)$ & $161(47,4)$ \\
\hline Sim & $94(27,6)$ & $61(17,9)$ & $24(7,1)$ & $179(52,6)$ \\
\hline Total & $159(46,8)$ & $112(32,9)$ & $69(20,3)$ & $340(100,0)$ \\
\hline
\end{tabular}

Fonte: Reis DA, Silva MA, 2019. 


\section{DISCUSSÃO}

No presente estudo, os participantes tiveram um predomínio na faixa etária entre 60 a 69 anos e com predominância do sexo feminino em relação ao masculino, resultado semelhante a pesquisa realizada em Guarapuava- Paraná, Dourado- Mato Grosso do Sul e Ituiutaba-Minas Gerais (PILGER C, et al., 2011; ALVARENGA MRM, et., 2011; MELO JV, et al., 2017).

Estudos demonstram que as mulheres são as que possuem maior longevidade, levando à ocorrência da chamada "feminização da velhice". Supõe-se que isso ocorra devido ao maior autocuidado que a mulher tem com relação a sua saúde, diferentemente dos homens, que não possuem o hábito de se cuidar, visto que, para eles, procurar pelo serviço de saúde é um sinal de fraqueza, vulnerabilidade e fragilidade, critérios esses que se enquadram em características femininas, o que implicaria em uma desconfiança da masculinidade, colocada pela imaginação da sociedade (LIMA CLJ, et., 2013; VICTOR FJ, et al., 2009). Os dados mostram também que a maioria dos idosos tem a cor parda, sendo um diferencial dos outros estudos nacionais que apresentam maioria dos idosos de cor branca (SILVA ALS, et al., 2009; CAMPOLINA AG, et al., 2013). Esse fato pode ser decorrência do estudo ser realizado na região norte do Brasil, especificamente na região amazônica, onde a grande maioria é de raça cabocla (descendentes de indígenas com branco). Conforme o IBGE (2011), em relação à declaração de cor e raça das regiões do Brasil, as regiões norte e nordeste possuíam em sua maioria a cor parda e regiões sul e sudeste possuíam sua maioria de cor branca.

Quanto ao estado conjugal, os participantes desta pesquisa eram casados ou mantinham uma união estável (191/56,8\%) e eram viúvos (85/25,0\%), corroborando os estudos de Silva ALS, et al. (2009) e Luz EP, et al. (2014), que apresentaram como maior frequência entre os idosos entrevistados, casados seguidos de viúvos. Entretanto, observou-se um diferencial no estudo revelado por Santos SR, et al. (2002), apresentando um padrão inverso, com $41 \%$ de viúvos e $39 \%$ de casados, em decorrência de os idosos estarem vivendo mais e tendo a oportunidade de passar mais tempo casados, e os que estão ficando viúvos, estão tendo oportunidade de se casar novamente, permitindo construir um novo relacionamento na terceira idade.

A baixa escolaridade prevaleceu, variando de analfabetos $(44,1 \%)$ ou um a cinco anos de estudos $(43,2 \%)$, corroborando com o estudo de Lima CLJ, et al. (2013), Silva ALS, et al. (2009), Campolina AG, et al. (2013).

Com relação à renda mensal da família, a maioria dos idosos recebiam entre dois a três salários mínimos. Esse fato limita o acesso a bens de serviços e de consumo, como alimentação, vestuário e moradia, além de se agravar quando as pesquisas corroboram que grande parte dos idosos são provedores financeiros de suas famílias (VICTOR JF, et al., 2013). Além disso, a principal fonte de renda para os idosos entrevistados foi aposentadoria, seguida do trabalho autônomo. E ainda, segundo Silva ALS, et al. (2009), destaca que a aposentadoria é a principal fonte de renda entre os idosos.

Um estudo realizado sobre o perfil epidemiológico na UBS do município de Cambé do Estado do Paraná, mostrou dados semelhantes, no qual $68 \%$ dos entrevistados apresentaram HAS e, desses dados, $91 \%$ eram mulheres (SILVA ALS, et al., 2009). A HAS é apontada como a principal doença crônica da velhice, principalmente entre idosos de 80 anos ou mais, além de contribuir para o surgimento de dificuldades nas AVDs e, consequentemente, interferência na capacidade funcional (LIMA CLJ, et al., 2013).

Estudos epidemiológicos têm demonstrado o aumento da prevalência da HAS com a idade avançada, sendo que sua ocorrência está associada aos fatores de riscos como estilo de vida e hábitos alimentares (CAMPOLINA AG, et al., 2013; PILGER C, et al., 2011).

Tal dado pode ser justificado através do estudo de Victor JF, et al. (2019), sobre o perfil socioeconômico e clínico em idosos, afirmando que a HAS é mais prevalente em mulheres, e em indivíduos de baixa renda e escolaridade, sendo que estas características estão presentes na pesquisa.

A HAS ocasiona transformações expressivas na vida dos indivíduos, seja na esfera psicológica, familiar, social ou econômica. Essas mudanças provocam rupturas no modo de viver, exigindo dos idosos as modificações dos seus hábitos diários, a fim, também, de não ocasionar novas doenças crônicas geradas pela enfermidade (LOPES MCL, et al., 2008). 
O estudo de Pilger C, et al. (2011), constatou a existência de 3,0\% dos entrevistados com dislipidemia entre idosos. Com relação à DM, na pesquisa desenvolvida por Silva ALS, et al. (2009), evidenciou-se que os idosos entrevistados eram 52\% de mulheres no grupo etário de 60 a 69 anos. O Ministério da Saúde refere que a DM associada, concomitantemente, com a HAS representa um grande fator de risco para 0 desenvolvimento de doenças coronarianas e para complicações microvasculares como a retinopatia e a nefropatia (BRASIL, 2013). A DM é uma doença crônica, que pode restringir nas atividades cotidianas dos indivíduos, devido às comorbidades que alguns idosos podem vir a apresentar, contribuindo dessa forma para a diminuição da autoestima e, portanto, afetando a qualidade de vida (CARDOSO MI, et al., 2011).

Com relação à prática para o autocuidado, os idosos realizavam de quatro a cinco refeições por dia. Esse pode ser explicado por questões culturais de realizar apenas refeições como café da manhã, almoço, lanche da tarde e o jantar, que aproxima dos hábitos amazonense. Na sua maioria, os idosos informaram que não têm o hábito de fumar e consumir bebida alcoólica, sendo representativo em todas as faixas etárias.

Nesta investigação, à prática de atividade física regular foi demonstrada pelos participantes do estudo quanto a realização do exercício físico. Estudo realizado na USF com uma amostra de 351 idosos no município Cambé-PR, demonstrou que apenas $29 \%$ dos entrevistados praticam algum tipo de atividade física (SILVA ALS, et al., 2009). Fato como esse é justificado pela falta de tempo e local para desenvolvimento das atividades além, também, da necessidade de implementação de política pública para o incentivo de atividades físicas no grupo da terceira idade.

É interessante apresentar algumas limitações desta pesquisa com referência ao universo e à amostra, uma vez que se limitou aos idosos cadastrados em UBS e residentes em áreas urbanas, consequentemente, não abrangendo toda a realidade dos idosos do município. Outras limitações são pela existência de pessoas que não são cadastradas nas UBS que são chamadas de "áreas descobertas" e vivem em locais onde determinam aspecto de suas vidas e saúde, realidades que mostrariam suas especificidades importantes.

\section{CONCLUSÃO}

O estudo possibilitou caracterizar a população idosa estudada como mulheres idosas jovens (faixa etária de 60 a 69 anos), cor parda, casadas, com baixo nível de escolaridade, com renda familiar mensal de dois a três salários mínimos. Evidenciou-se que as DCNTs com maior frequência foram: HAS, Dislipidemia e DM. Quanto às práticas de autocuidado, os respondentes demonstraram que fazem de quatro a cinco refeições diárias, não têm o hábito de tabagismo e etilismo, além de praticar atividade física regulamente numa frequência de três a quatro vezes por semana. Esses dados revela a importância dos profissionais integrantes da equipe ESF conhecerem as peculiaridades que envolvem a pessoa idosa da sua área de abrangência com o intuito de terem bases concretas para planejamento de ações em saúde a partir das necessidades desses indivíduos.

\section{FINANCIAMENTO}

Fundação de Amparo e Pesquisa do Estado do Amazonas (FAPEAM). *Trabalho desenvolvido pelo Programa Institucional de Bolsa de Iniciação Científica (PIBIC) da Universidade Federal do Amazonas, com o financiamento da Fundação de Amparo à Pesquisa do Estado do Amazonas (FAPEAM). Parte integrante da pesquisa: "Perfil de Doenças Crônicas Não Transmissíveis em idosos pertencentes à rede de atenção Básica do município de Coari, Amazonas", aprovado pelo Comitê de Ética em Pesquisa com Seres Humanos da Universidade Federal do Amazonas através do parecer nำ786/2014.

\section{REFERÊNCIAS}

1. ALVARENGA MRM, et al. Perfil social e funcional de idosos assistidos pela estratégia da saúde da Família. Cogitare Enferm, 2011; 16(3): 478-85.

2. BRASIL. Ministério da Saúde. Secretária de Atenção à Saúde. Estratégia para o cuidado da pessoa com doença crônica: diabetes mellitus. Brasília: Ministério da Saúde, 2013. 
3. BRASIL. Ministério da Saúde. Conselho Nacional de Saúde. Resolução n. 466, de 12 de dezembro de 2012. Aprova diretrizes e normas regulamentadoras de pesquisa envolvendo seres humanos. Brasília: Conselho Nacional de Saúde, 2012.

4. CAMPOLINA AG, et al. A transição de saúde e as mudanças na expectativa de vida saudável da população idosa: possíveis impactos da prevenção de doenças crônicas. Cad Saúde Pública, Rio de Janeiro, 2013; 29(6): $1217-1229$.

5. CARDOSO MI, et al. Práticas de autocuidado desenvolvidas por diabéticos tipo 2 em uma unidade de saúde da família. Revista Eletrônica Gestão \& Saúde, 2011; 02(01): 277-290.

6. CHEEVER KH, BRUNNER LS, SUDDARTH DS. Tratado de Enfermagem Médica-Cirúrgica. 13. ed. Rio de Janeiro: Guanabara Koogan, 2016.

7. DUCAN BB, et al. Doenças Crônicas Não Transmissíveis no Brasil: prioridade para enfrentamento e investigação. Rev Saúde Pública, 2012;46(Supl):126-34.

8. FIGUEIREDO AH. Brasil: uma visão geográfica e ambiental no início do século XXI. Rio de Janeiro: Instituto Brasileiro de Geografia e Estatística, Coordenação de Geografia, 2016.

9. Instituto Brasileiro de Geografia e Estatística. Coordenação de População e Indicadores Sociais. Síntese de indicadores sociais: uma análise das condições de vida da população brasileira. Rio de Janeiro: Instituto Brasileiro de Geografia e Estatística, Coordenação de Geografia, 2016.

10. LIMA CLJ, et al. Perfil sociodemográfico e clínico de idosos institucionalizados. Rev enferma UFPE on line, Recife, 2013; 7(10): 6027-34.

11. LUZ EP, et al. Perfil sociodemográfico e de hábitos de vida da população idosa de um município da região norte do Rio Grande do Sul, Brasil. Rev Bras Geriatr Gerontol, Rio de Janeiro, 2014; 17(2):303-314.

12. LOPES MCL, et al. O autocuidado em indivíduos com hipertensão arterial: um estudo bibliográfico. Rev. Eletr. Enf, 2008;10(1):198-211.

13. MALTA DC, et al. Doenças crônicas não transmissíveis e a utilização de serviços de saúde: análise da Pesquisa Nacional de Saúde no Brasil. Rev Saúde Publica, 2017; 51:1s-10s.

14. MELO JV, et al. Perfil de Saúde dos idosos atendidos nas Unidades Básicas de Saúde da Família (UBSF) em Ituiutaba, Minas Gerais. Rev Aten Saúde, 2017; 15(53): 66-75.

15. PILGER C, et al. Características sociodemográficas e de saúde de idosos: contribuições para os serviços de saúde. Rev. Latino-Am. Enfermagem Rev Latino-Am. Enfermagem, 2011 Oct; 19(5): 1230-1238.

16. ROCHA FCV, et al. O cuidado do enfermeiro ao idoso na estratégia saúde da família. Rev. Enferm.UERJ, 2011; 19(2):189-91.

17. SANTOS SR, et al. Qualidade de vida do idoso na comunidade: aplicação da escala de flanagan. Rev. Latino- Am. Enfermagem, 2002; 10(6): 757-764.

18. SILVA ALS, et al. Perfil epidemiológico dos idosos de uma unidade de saúde da Família. UNOPAR Cient, 2009; $11(2): 27-33$.

19. VICTOR JF, et al. Perfil sociodemográfico e clínico de idosos atendidos em Unidade Básica de Saúde da Família. Acta Paul Enferm, 2009; 22(1):49-54. 